\title{
Cylindrical Dielectric Resonator Antenna for SHF Band Application
}

\author{
Ahmed Benomar ${ }^{1}$, Noureddine Boukli Hacene ${ }^{2}$, Hicham Megnafi ${ }^{3} \&$ Patrick Vaudon $^{4}$ \\ ${ }^{1,2,3}$ LTT Laboratory, telecommunication dept, Univ. Abou Bakr Belkaid, Tlemcen, Algeria \\ ${ }^{4}$ XLIM Laboratory,OSA dep, Univ. of Limoges, France \\ Correspondence: Ahmed Benomar, LTT Laboratory, telecommunication dept Univ. Abou Bakr Belkaid B.P N ${ }^{\circ}$ \\ 119, Tlemcen, Algeria. E-mail: benomar.sa@gmail.com
}

Received: December 12, 2012 Accepted: February 21, 2013 Online Published: March 30, 2013

doi:10.5539/nct.v2n1p28 URL: http://dx.doi.org/10.5539/nct.v2n1p28

\begin{abstract}
In this paper, an embedded cylindrical dielectric resonator antenna (CDRA) is presented . The antenna, excited by a coaxial feeder is composed of one host cylinder made from ceramic, with a constant $\varepsilon_{\mathrm{r} 1}=36.7$ operating at a frequency of $1.96 \mathrm{GHz}$; which excites the $\mathrm{HEM}_{11 \delta}$ mode and the embedded cylindrical element with radius $\mathrm{a}_{2}$ and permittivity $\varepsilon_{\mathrm{r} 2}$.

In order to get a wideband antenna, that can offer multi-resonant frequencies used for SHF band, parameters retunes on the radius and permittivity of additional element are presented. The study shows briefly, broadband dielectric resonator antennas (DRAs) design using HFSS simulator tool, based on finite element method.
\end{abstract}

Keywords: CDRA, bandwidth, permittivity, return loss, coaxial probe, dimensions

\section{Introduction}

In recent years, a cylindrical dielectric resonator antenna (CDRA) excited by a coaxial probe proposed by Long, McAllister and Shen (1983) has received great deal of attention in various applications such as armored filters or oscillators, for their advantageous small size, low loss, low cost and attractive features in terms of high radiation efficiency as it has negligible metallic loss, and high Q factor (Kajfez \& Guillon, 1986; Long, McAllister, \& Shen, 1983; Petosa, Ittipiboon, Antar, \& Roscoe, 1998; Luk \& Leung, 2002). Indeed, when a resonator is placed in a cavity, it presents a high quality factor. This allows the production of a highly selective filter; in other hand the demand on wireless mobile communications has led to the development of a different geometries of the antenna such as rectangular, cylindrical, hemispherical, circular, triangular etc. are possible. The resonant frequency of the DRA is predominantly determined by its size, shape radius, height ...) and material permittivity $\left(\varepsilon_{\mathrm{r}}\right)$ (Kajfez \& Guillon, 1986).

Moreover, various antenna features such as bandwidth, input impedance, and radiation patterns can easily be regulated by varying the antenna specifications and feed mechanisms.

In the present work, a simple cylindrical dielectric resonator antenna as shown in Figure 1 is extensively investigated as suitable antenna for wireless applications; The CDRA is excited by a coaxial probe.

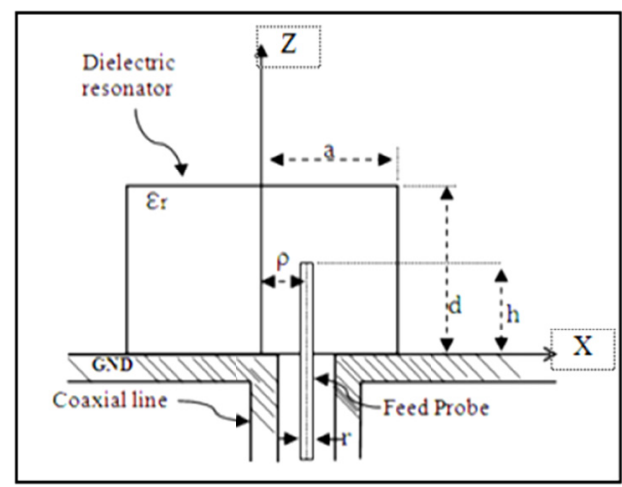

(a)

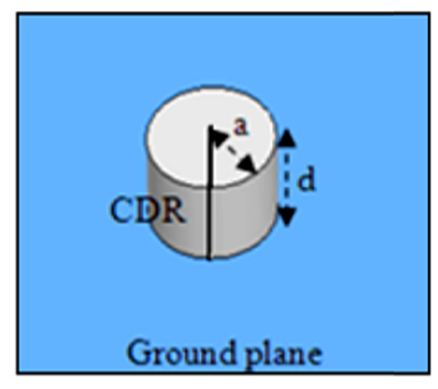

(b)

Figure 1. DRA configuration (a) Side view; (b) 3-D view 
The radius and the constant permittivity of additional cylindrical are varied in order to observe how the path loss, and the bandwidth of CDRA are affected and in mean time to get the optimum of these values, while preserving the radiation characteristics of the dominant mode (Chair, Kishk, \& Lee, 2007; Kishk, Auda, \& Ahn, 1989).

\section{Method}

\subsection{CDRA Theory}

The preferable mode for broadside radiation is the $\mathrm{HEM}_{11 \delta}$ mode, and for filter designs the mode $\mathrm{TE}_{01 \delta}$ because of its high $\mathrm{Q}$ factor performance, while for dual band radiating purposes the $\mathrm{TM}_{01 \delta}$ mode is chosen for its Omni-directional pattern (Kajfez \& Guillon, 1986).

The cylindrical resonator antenna under investigation has a height and radius $d$ and a, respectively. The ground plane, assumed to be of finite extent, supports the dielectric cylinder. The simple way of excitation of the lowest mode of the structure which is the HEM mode is by using a coaxial probe, vertically orientated with height equal to $h$ and located at distance equal to $\rho$ far from the centre of DRA on phi $=0^{\circ}$ as on Figure 1 .

The characteristics of the CDRA can be divided into two parts, resonate frequency and field distribution:

\subsubsection{Resonant Frequency}

The resonant frequency of the cylindrical DRA was calculated with (1) described in Kajfez and Guillon (1986). This gives a frequency of $1.96 \mathrm{GHz}$ for the single CDRA. In practical applications, we are interested in the fundamental (dominant) mode, which has the lowest resonant frequency. It has been found that the fundamental mode is the $\mathrm{HEM}_{110}$ mode. The resonant frequency given by Equation (1) is in function of:

- Dielectric constant " $\varepsilon_{\mathrm{r}}$ '

- Radius "a"

- Height "d"

- $\mathrm{C}$ is the velocity of light in free space

$$
. \mathrm{f}=\left(2.208 \times \mathrm{C} / 2 \pi \times \mathrm{d} \sqrt{\varepsilon_{\mathrm{r}} \pm 1}\right)\left[1+0.7013 \frac{\mathrm{a}}{\mathrm{b}}-0.002718\left(\frac{\mathrm{a}}{\mathrm{b}}\right)^{2}\right]
$$

The frequency obtained by the Eigen mode solver of HFSS simulator is very close to the value obtained by Equation (1) as on Table 1.

Table 1. Frequency got by simulator

\begin{tabular}{ccc}
\hline Configuration antenna & Freq (Eigen mode solver) & Permittivity \\
\hline Single DRA & $1.94 \mathrm{GHz}$ & 36.7 \\
\hline
\end{tabular}

\subsubsection{Field Distributions}

The filed distribution of antenna, based on a cylindrical dielectrics resonator looks like the dipole when the radiations of low fundamental modes are in the cavity. In mean time the adjustment of antenna shape, size or alimentation position could maximize the bandwidth and change the antenna behavior to multi-mode antenna (Kajfez \& Guillon, 1986; Kishk, Auda, \& Ahn, 1989).

The field pattern inside the antenna of the mode $\mathrm{TM}_{01 \delta}$ shows behavior of a quarter-wavelength monopole and for the mode $\mathrm{HEM}_{11 \delta}$ mode looks ideally like half-wave dipole. The field line appears to be generated by magnetic dipole with a dipole axis located horizontally, on the equatorial plan of CDR (Kajfez \& Guillon, 1986). During the simulation, more than one mode may be excited by the feeding mechanism that affects the radiation pattern trend. Furthermore, the ground plane will be fixed as finite dimension (Kajfez \& Guillon, 1986; Kishk, Auda, \& Ahn, 1989). On the numerical simulation, the effects are taken in a consideration.

\section{Principles of Investigation}

\subsection{CDRA Composed by Single Cylinder}

Under the HFSS simulator the proposal CDRA as depicted on Figure 2 is composed by only single ceramic cylinder with the following characteristics: permittivity $=36.7$, radius $a_{1}=12.65 \mathrm{~mm}$ and height $d_{1}=9.6 \mathrm{~mm}$ that $\mathrm{d} / \mathrm{a}=0.75<1$ ). The CDRA is fed energy by a $50 \Omega$ Coaxial line with width $\mathrm{r}=0.65 \mathrm{~mm}$, length $\mathrm{h}=5.5 \mathrm{~mm}$ at position $\rho=11 \mathrm{~mm}$ and over a ground plan of $75 \times 75 \mathrm{~mm}$. 


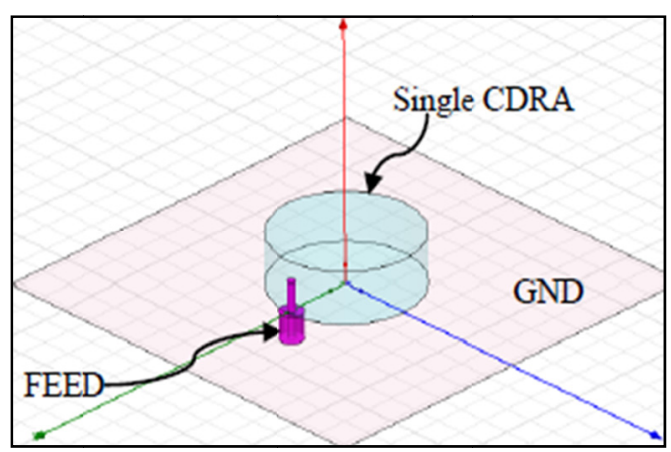

Figure 2. Geometry of the probe-fed CDRA

The simulation result on both figures "Figure 3 and Figure 4" demonstrates that CDRA for HEM 1ld $_{\text {mode }}$ is shows good agreement in term of resonant frequency which is equal to $1.91 \mathrm{GHz}$ very close to the frequency obtained in Equation (1) and the Eigen mode solver, otherwise the reflection coefficient $S_{11}=-50 \mathrm{~dB}$ shows a good adaptation of the antenna.

The radiation pattern inside the antenna E_phi /E_theta has a broadside direction and a linearly polarized radiation across two orthogonal planes (E and $\mathrm{H}$ planes) by using a suitable feed position, and good probe length, the radiation patterns are nearly ideal because some higher order modes, with non-broadside radiation patterns, are excited and disturb the radiation pattern axial symmetry (Kishk, Auda, \& Ahn, 1989; Junker, Kishk, \& Glisson, 1994).

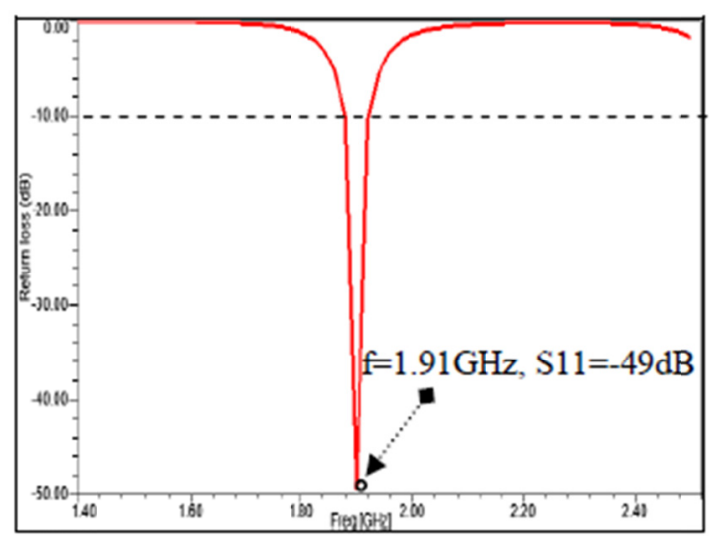

Figure 3. Computed return losses of CDRA against frequency a $=12.65 \mathrm{~mm} \mathrm{~d}=9.6 \mathrm{~mm}$, $\rho=11.75 \mathrm{~mm}, \mathrm{~h}=5.5 \mathrm{~mm}, \mathrm{r}=0.65 \mathrm{~mm}, \mathrm{GND}=75 \times 75 \mathrm{~mm}$

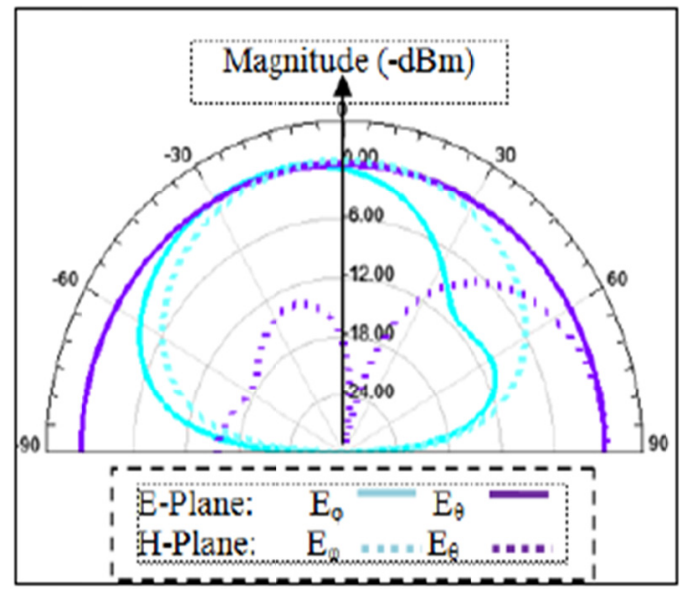

Figure 4. Simulated radiation patterns of the single CDRA 


\subsection{CDRA Composed by Two Cylinders}

In this part we use the same antenna as describe on part 1 and by adding a second cylinder to the first cylinder, the CDRA become composed of two cylinders as shown in Figure 5.

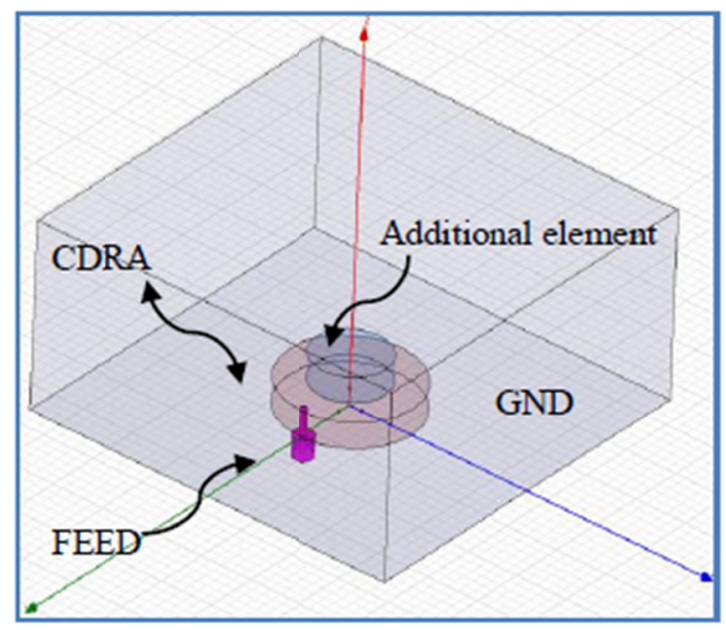

Figure 5. Geometry of the embedded CDRA

From Equation (1) we notice that the frequency is highly affected by the variation of the dimension of the cylinder and the permittivity of dielectric, indeed, the addition of a second cylinder increases the permittivity of the system and also the dimension too. The embedded cylinder element with radius $a_{2}=x \mathrm{~mm}$, height $d_{2}=5.6 \mathrm{~mm}$, and permittivity $\varepsilon_{\mathrm{r}}=\mathrm{y}$. The following will be divided into sub-part one for radius effect and the second for permittivity effect by varying the permittivity of the second cylinder based on the chose of optimized radius.

\subsubsection{Effect of Varying the Radius of Additional Element}

The radius of the second cylinder $\mathrm{a}_{2}$ will be changed from 2 to $11 \mathrm{~mm}$, the permittivity is kept equal to $\varepsilon_{\mathrm{r}}=10$ (a low value to reduce the impact of the permittivity of the system on the characteristics of the antenna as on Equation (1)). The computed return loss $S_{11}$ is plotted against the frequency in Figure 6 for different values of $a_{2}$ from (Huang \& Kishk, 2007; Chair, Kishk, \& Lee, 2007; Buerkle, Sarabandi, \& Mosallaie, 2005; Kishk, Ahn, \& Kajfez, 1989; Tam \& Murch, 1999). It is found that as a2 increases, the return loss decreases and the bandwidth becomes narrow, so the radius should be tacked to make a compromise between the adaptation and the bandwidth. Furthermore, if the radius is very small, the impedance becomes highly capacitive, which should normally be avoided.

Choosing the Radius $\mathrm{a}_{2} \approx \mathrm{a}_{1} / 2$ (it is not rule it depends on the geometry of the antenna structure) of the additional CDRA maximizes the excitation of the dominant mode (Kishk, Auda, \& Ahn, 1989; Junker, Kishk, \& Glisson, 1994).

The measured bandwidths for four different value of $\mathrm{a}_{2}$ are also tabulated in Table 2 It is seen that the antenna bandwidth is higher for $\rho=7 \mathrm{~mm}$ (From this result we keep $\mathrm{a}_{2}=7 \mathrm{~mm}$ for the next part) (Junker, Kishk, \& Glisson, 1994).

Table 2. Bandwidth against frequencies

\begin{tabular}{ccc}
\hline Radius $\mathrm{a}_{2}(\mathrm{~mm})$ & Frequency $(\mathrm{GHZ})$ & Bandwidth $(\mathrm{MHZ})$ \\
\hline 2 & 7.78 & $\mathrm{X}$ \\
4 & 7.96 & 90 \\
7 & 7.75 & 400 \\
\hline
\end{tabular}




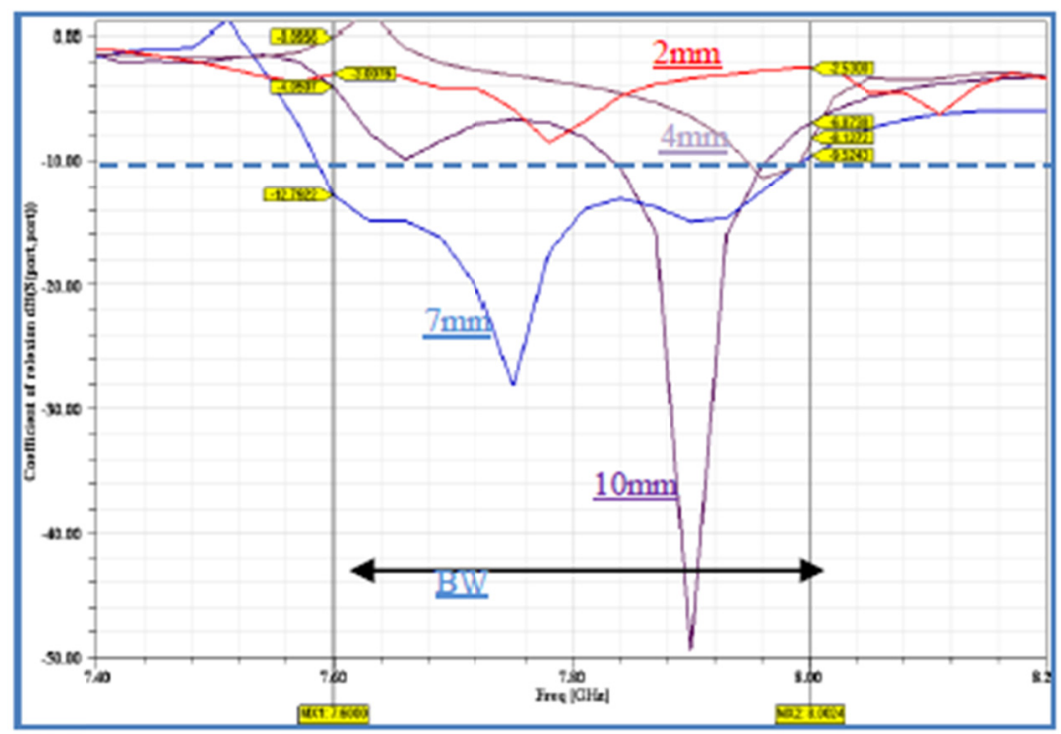

Figure 6. Effect of varying Radius a2 from $2 \mathrm{~mm}$ to $11 \mathrm{~mm}$

\subsubsection{Effect of Varying the Permittivity of the Additional Element}

From the above study the radius $\mathrm{a}_{2}$ of the additional cylinder which most affected the CDRA characteristics is equal to $7 \mathrm{~mm}$ at this value the permittivity $\varepsilon_{\mathrm{r} 2}$ of the second cylinder is varied from 10 to 90 , We notice that by changing $\varepsilon_{\mathrm{r} 2}$ the return loss $\mathrm{S}_{11}$ and antenna bandwidth change inversely, as shown on Figure 7 and Table 3 for different (Huang \& Kishk, 2007; Chair, Kishk, \& Lee, 2007; Buerkle, Sarabandi, \& Mosallaie, 2005).

Table 3. Bandwidth against frequencies

\begin{tabular}{ccc}
\hline Permittivity & Frequency (GHZ) From-To & Bandwidth (MHZ) \\
\hline 40 & $6.94-7.25$ & 210 \\
70 & $6.80-7.20$ & 900 \\
80 & $6.89-7.16$ & 400 \\
\hline
\end{tabular}

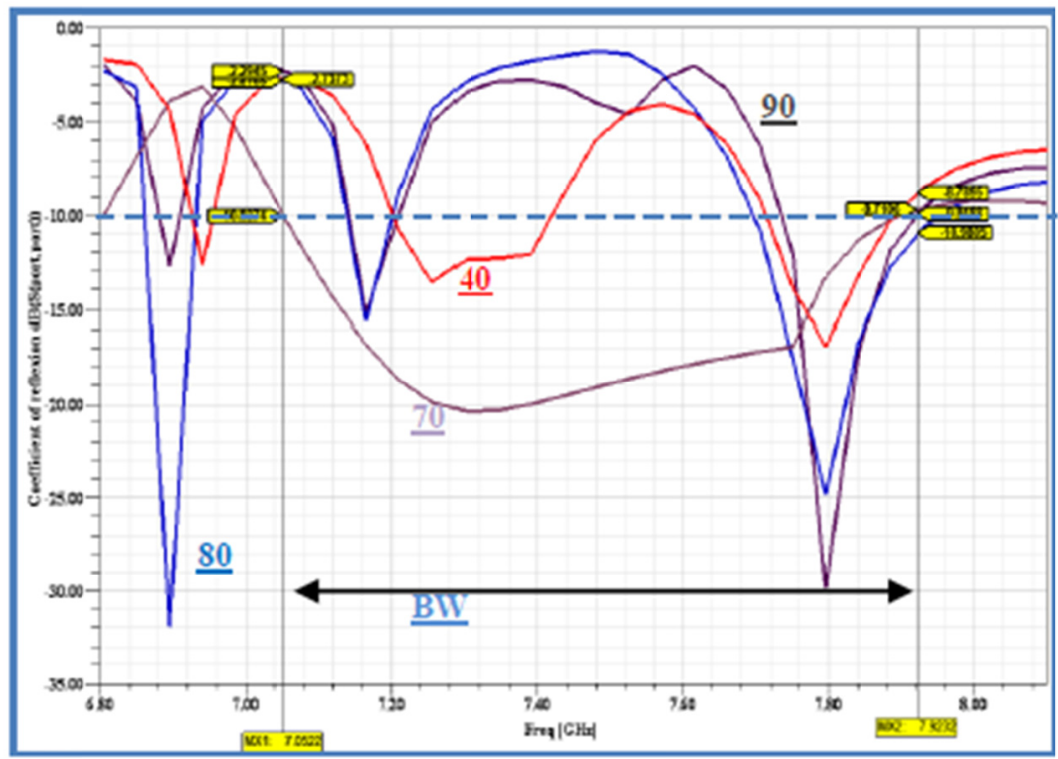

Figure 7. Effect of varying Additional element permittivity $\left(\varepsilon_{\mathrm{r} 2}\right)$ from 10 to 90 
From Table 3 it is observed that the impedance bandwidth (return loss $10 \mathrm{~dB}$ ) increases when $\varepsilon_{\mathrm{r} 2}$ increase. The computed impedance bandwidths for $\varepsilon_{\mathrm{r} 2}=70$ it the more large at $900 \mathrm{MHz}$.

Otherwise by increasing the permittivity of the CDRA new antenna behavior appear as multi- frequencies mode over SFH band.

\section{Conclusion}

In this paper we have presented one of many approaches which using finite element methods by using HFSS simulator tools, the effect on resonant frequency and return loss by changing dimensions of the antenna in terms of radius, height and permittivity of the cylinder were studied.

For suitable application it is recommended that the choice of permittivity and the dimension of the antenna be considered carefully because they have a great affect on the characteristics in terms of bandwidth and resonant frequency.

To achieve large band and multimode antenna behavior, the feeding mechanism should be adjusted into suitable position near the edge of the cylinder, and the ratio of the radius over height should be minimal.

The concept of having Multi band antenna has been achieved by a superposed two-cylinder structure, furthermore resonances frequencies have been reduced compared to the single structure and good performances have been obtained on the bandwidth.

The proposed antenna can be used for SUH applications with a wide bandwidth $(\approx 1 \mathrm{GHz})$ and provides good efficiency.

\section{References}

Buerkle, A., Sarabandi, K., \& Mosallaie, H. (2005). Compact slot and dielectric resonator antenna with dual-resonance, broadband characteristics. IEEE Trans. Antennas Propag, 53(3), 1020-1027. http://dx.doi.org/10.1109/TAP.2004.842681

Chair, R. C., Kishk, A. A., \& Lee, K. F. (2007a). Low profile wideband embedded dielectric resonator antenna, Microw. Antennas Propag., 1(2), 294-298. http://dx.doi.org/10.1049/iet-map:20060028

Chair, R. C., Kishk, A. A., \& Lee, K. F. (2007b). Wideband stair-shaped dielectric resonator antennas, Microw. Antennas Propag., 1(2), 299-305. http://dx.doi.org/10.1049/iet-map:20060029

Huang, W., \& Kishk, A. (2007). Compact wideband multi-layer cylindrical dielectric resonator antenna, Microw. Antennas Propag., 1(5), 998-1005. http://dx.doi.org/10.1049/iet-map:20070028

Junker, G. P., Kishk, A. A., \& Glisson, A. W. (1994). Input impedance of dielectric resonator antennas excited by a coaxial probe. IEEE Trans. Antennas Propagat, 42, 960-966. http://dx.doi.org/10.1109/8.299598

Kajfez, D., \& Guillon, P. (1986). Dielectric Resonators. Norwood, MA: Artech House.

Kishk, A. A., Ahn, B., \& Kajfez, D. (1989). Broadband stacked dielectric resonator antennas. Electron. Lett., 25(18), 1232-1233. http://dx.doi.org/10.1049/el:19890826

Kishk, A. A., Auda, H. A., \& Ahn, B. C. (1989). Radiation characteristics of cylindrical dielectric resonator antenna with new applications. IEEE Trans. Antennas Propagat. Soc. Newsletter, 31, 7-16.

Long, S. A., McAllister, M. W., \& Shen, L. C. (1983). The resonant cylindrical dielectric cavity antenna. IEEE Trans. Antennas Propag., 31(3), 406-412. http://dx.doi.org/10.1109/TAP.1983.1143080

Luk, K. M., \& Leung, K. W. (2002). Dielectric resonator antennas, ser. Electronic \& Electrical Engineering Research. Baldock, U.K.: Research Studies.

Petosa, A., Ittipiboon, A., Antar, Y. M. M., \& Roscoe, D. (1998). Recent advancesin dielectric-resonator antenna technology. IEEE Antennas Propag. Mag., 40(3), 35-48. http://dx.doi.org/10.1109/74.706069

Tam, M. T. K., \& Murch, R. D. (1999). Compact circular sector and annular sector dielectric resonator antennas. IEEE Trans. Antennas Propag., 47(5), 837-842. http://dx.doi.org/10.1109/8.774138 